\title{
Piezoresistive Miniaturkraftsensoren in LTCC
}

\author{
Christian Lenz, Steffen Ziesche, Uwe Partsch
}

Fraunhofer Institut für keramische Technologien und Systeme (IKTS), Dresden (01277), Winterbergstraße 28

\section{Zusammenfassung}

In dieser Arbeit präsentieren wir die neuesten Entwicklungen von piezoresistiven Miniaturkraftsensoren mit verschiedenen Verformungskörpern in LTCC (Low Temperature Cofired Ceramic) und siebgedruckten Dickschichtwiderständen am Fraunhofer IKTS. Dazu wurden verschiedene Sensorvarianten konzipiert, hergestellt und hinsichtlich ihrer Eigenschaften untersucht. Der Fokus ist bei der Entwicklung besonders auf eine hohe Genauigkeit, eine kosteneffiziente Fertigung durch Miniaturisierung und die Sensorhausung gelegt. Einige wichtige Entwicklungsschritte und Ergebnisse werden hier vorgestellt.

\section{Einleitung}

Die LTCC-Technologie ist neben ihrer eigentlichen Funktion als Schaltungsträger auch als Integrationsplattform für Mikrosysteme mit sensorischen, aktorischen und fluidischen Komponenten geeignet. Das Stapeln und Laminieren verschieden strukturierter Keramikfolien erlaubt es komplexe dreidimensionale Strukturen (z. B. Membranen, Balken, Kavitäten und Kanäle) zu integrieren.

Die LTCC-Technologie kam bereits in verschiedenen Sensoren für mechanische Größen (Druck, Beschleunigung, Kraft) zur Anwendung und zeigte positive Eigenschaften hinsichtlich ihres linearen und nahezu hysteresefreien Materialverhaltens, ihrer Langzeitstabilität und der Reproduzierbarkeit [1-4]. Typischerweise werden dabei die Deformationen geeigneter Verformungskörper über das piezoelektrische oder piezoresistive Messprinzip detektiert. Beide lassen sich gut durch entsprechende Funktionsschichten im LTCC-Substrat umsetzen.

In diesem Bericht werden piezoresistive Miniaturkraftsensoren in LTCC vorgestellt, die im Gegensatz zu [3-4] auf Zug- und Druckrichtung ausgelegt sind. Design und Packaging sind dem kommerzieller Miniaturkraftsensoren für kleine Kräfte angelehnt. Durch den Vorteil der Nutzenfertigung, den diese Technologie bietet, kann der Sensor kostengünstig gefertigt werden. Weiterhin werden die wichtigsten Kenngrößen vorgestellt.

\section{Piezoresistives Messprinzip}

Kraftsensoren messen immer die Wirkung der Kraft auf einen Verformungskörper. Diese Belastung bewirkt Spannungen und Verformungen in ihm. Für die Wandlung dieser Wirkungen in ein elektrisches Sensorsignal eignet sich das piezoresistive Messprinzip. Dieses zeichnet sich durch eine hohe Genauigkeit, Langzeitstabilität und eine vielseitige Anwendbarkeit aus [5-6].

In diesem Fall wandelt ein dehnungsempfindlicher Dickschichtwiderstand die auftretenden Dehnungen in eine Widerstandsänderung um. Als Maß für die Abhängigkeit zwischen der Widerstandsänderung und der Dehnung ist der Gauge-Faktor $K$ definiert. Dieser ist material- und temperaturabhängig und kann je nach Belastungsrichtung (längs, quer) abweichen. Die Widerstandsänderung äußert sich schließlich in einer Wheatstone Brücke durch eine Änderung der Brückenspannung $U_{S}$ - dem Sensorsignal. Die Wirkungskette in Bild 1 verdeutlicht dieses Prinzip. 


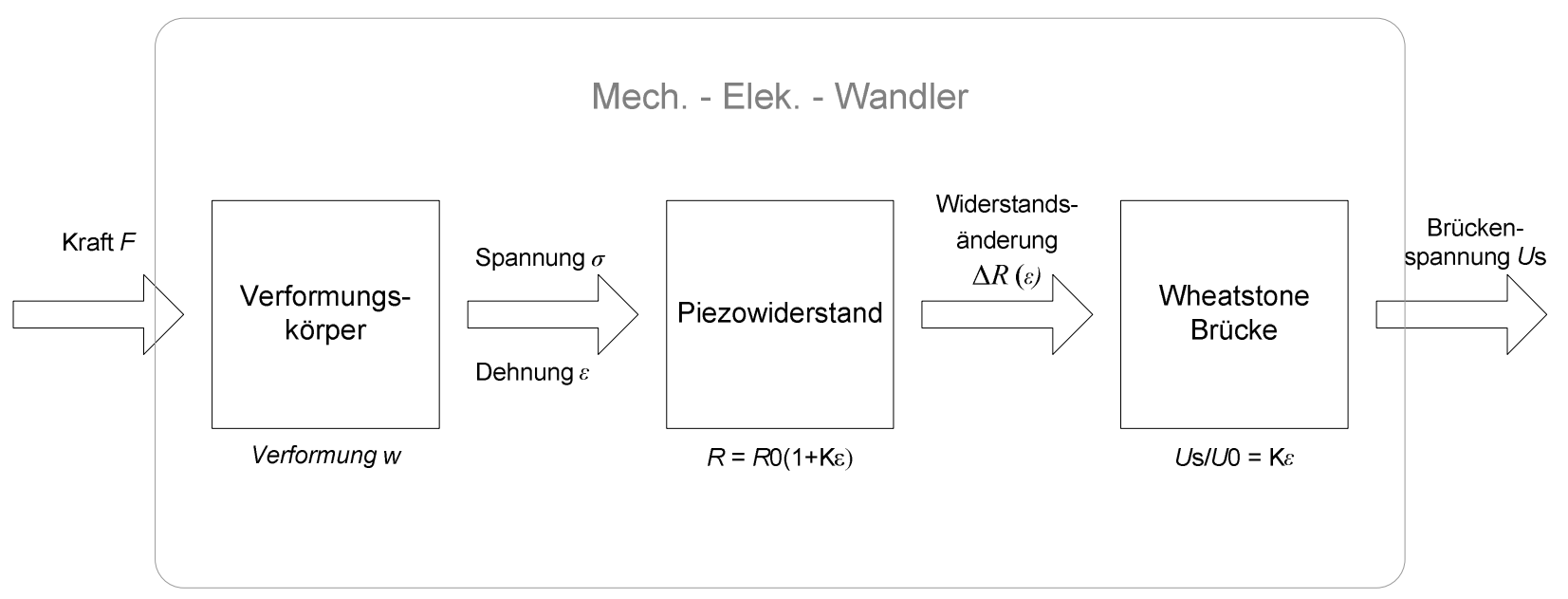

Bild 1 Wirkungskette eines piezoresistiven Kraftsensors im einachsigen Spannungszustand

\section{Sensoraufbau}

Die hier vorgestellten Sensoren sind im Standard-LTCC-Prozess konzipiert. Als Basismaterial kommt die Grünfolie GT951 von DuPont zur Anwendung. Die piezoresistiven Dickschichtwiderstände werden im kostengünstigen Siebdruckverfahren mit einer kommerziellen Paste prozessiert. Das Stapeln der verschieden strukturierten Folien ermöglicht den dreidimensionalen Aufbau mit vergrabenen oder verdeckten freistehenden Strukturen. Mit dem Sintern des Stapelverbundes entsteht ein quasi-monolithischer Aufbau.

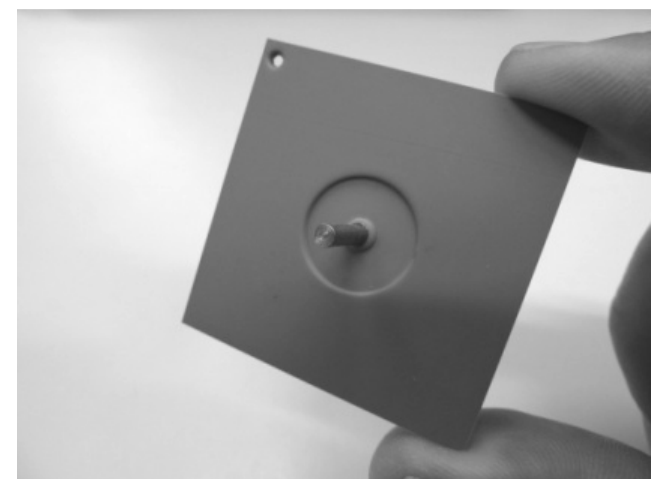

a)

Bild 2 Piezoresistive Miniaturkraftsensoren: a) Kraftsensor mit Kreisringplatte als Verformungskörper $\left(F_{\mathrm{N}}=5 \mathrm{~N}\right)$,

b) Kraftsensor mit Balkenelementen in Wagenradstruktur als Verformungskörper $\left(F_{\mathrm{N}}=1 \mathrm{~N}\right)-$ Schnittansicht, die oberste Lage ist nicht sichtbar

Am IKTS werden Miniaturkraftsensoren mit verschiedenen Verformungskörpern entwickelt. In der ersten Sensorgeneration ist das kraftempfindliche Element als Membran mit biegesteifen Zentrum (Kreisringplatte) für einen Nennkraftbereich $F_{\mathrm{N}}=5 \mathrm{~N}$ ausgeführt (Bild 2a). Während die Strukturierung der Geometrie vollständig im Grünzustand der LTCC erfolgt, werden die elektrischen Funktionsschichten erst nach dem Sinterprozess auf die Rückseite der Membran gedruckt (post-firing).

Die aktuelle Generation in Bild $2 \mathbf{b}$ besitzt vier Biegebalken, die über ein biegesteifes Zentrum zu einer Wagenradstruktur verbunden sind, als Verformungskörper $\left(F_{\mathrm{N}}=1 \mathrm{~N}\right)$. Hier finden sowohl die Strukturierungs- als auch die Druckschritte vollständig im Grünzustand statt. Dadurch kann das kraftempfindliche Element im Substrat vergraben werden.

Die Krafteinleitung bei beiden Varianten erfolgt über einen Gewindestift der auf dem biegesteifen Zentrum befestigt ist. Den Kraftanschlag erzeugt entweder ein weiterer Gewindestift oder auf der Substratrückseite befindliche Lötbumps (Bild 3a). 
Großer Vorteil der LTCC-Technologie ist Möglichkeit der automatisierten Fertigung im Nutzen. Bei der kleinen Bauform des aktuellen Sensors können somit 25 Sensoren je $\square 4$ Zoll-Substrat gleichzeitig gefertigt werden (Bild 3b). Zudem ermöglicht es der Mehrlagenaufbau die Sensoren leicht für andere Nennkraftbereiche auszulegen, indem die Verformungskörper aus anderen Folienkombinationen gebildet werden - bei gleichem Layout.

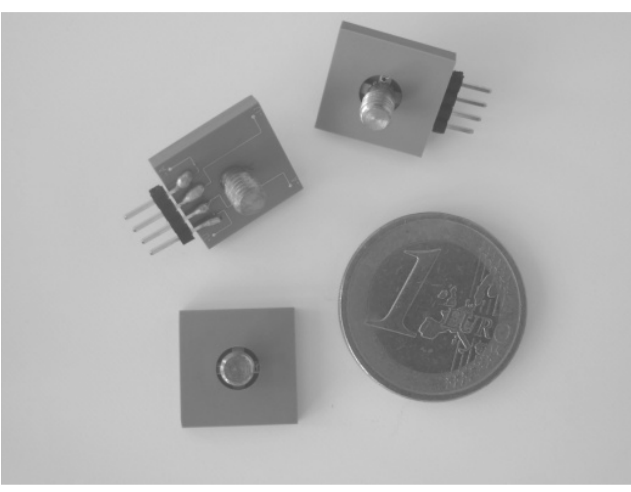

a)

Bild 3 Kraftsensor mit Wagenradstruktur: a) Sensoren mit Krafteinleitung und Kontaktierungen; b) Fertigung im Nutzen

\section{Sensoreigenschaften}

Die gefertigten Prototypen des Kraftsensors mit Kreisringplatte wurden umfangreich hinsichtlich mechanischer und elektrischer Kenngroßen untersucht. Sie zeichnen sich durch eine ansprechende Sensitivität von $\mathrm{S}=1 \mathrm{mV} /(\mathrm{V} \cdot \mathrm{N})$ bei guter Linearität $(\mathrm{L} \leq 0,22 \% \mathrm{FS})$ aus (Bild 4). Weiterhin weist die Kreisringplatte eine Querbruchkraft von $F_{\mathrm{B}}=70 \% F_{\mathrm{N}}$ auf bei einer geringen Querkraftempfindlichkeit von $S_{\mathrm{q}}=0,09 \mathrm{mV} /(\mathrm{V} \cdot \mathrm{N})$. Aufgrund des Aufbaus sind allerdings signifikante Unterschiede in Zug- und Druckrichtung zu verzeichnen.

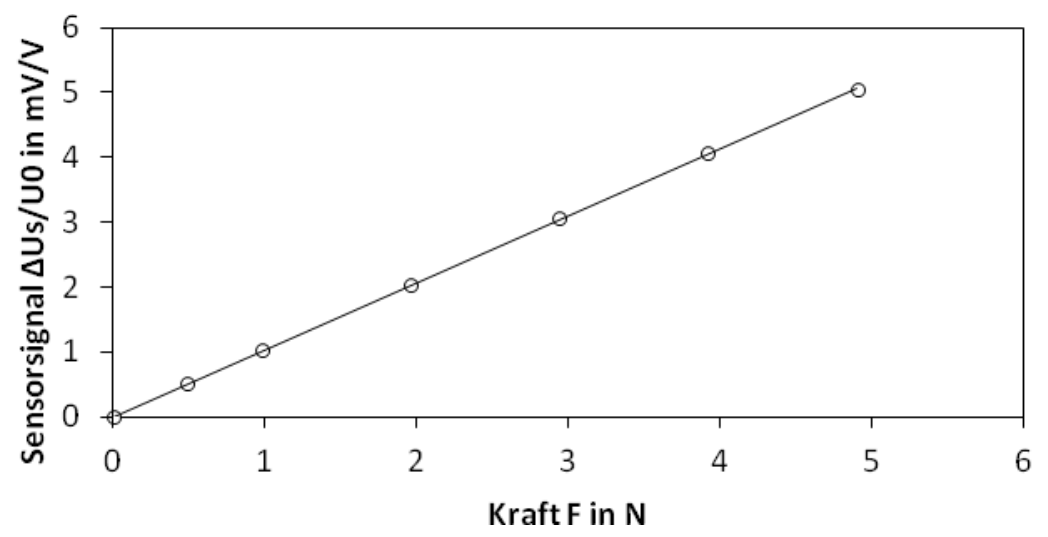

Bild 4 Messtechnisch ermittelte Kennlinie der Kreisringplatte ( $\mathrm{U}_{0}$ - Versorgungsspannung)

Die Ausführung der Wagenradstruktur mit dem vergrabenen Verformungskörper ermöglicht eine symmetrische Einspannung der Balkenelemente. Dadurch sind keine Unterschiede zwischen den beiden Kraftrichtungen zu erwarten. Die vollständige Charakterisierung dieser Sensorvariante ist Gegenstand weiterer Arbeiten. Vorerst können nur rechnerisch ermittelte Kenngrößen angegeben werden. Die Erfahrungen aus der Kreisringplatte zeigen jedoch, dass diese in guter Übereinstimmung zu den tatsächlichen Werten stehen. Tafel 1 fasst die wichtigsten Kenngrößen der beiden Sensorvarianten zusammen. 
Tafel 1 Eigenschaften der gefertigten Kraftsensoren

\begin{tabular}{|c|c|c|}
\hline Kenngrößen & Kreisringplatte & Wagenradstruktur \\
\hline Abmessungen in $\mathrm{mm}^{3}$ & $42 \times 42 \times 2$ & $14 \times 14 \times 2$ \\
\hline Nennkraft $F_{\mathrm{N}}$ in N & 5 & 1 \\
\hline Sensitivität $S$ in $\mathrm{mV} /(\mathrm{VN})$ & 1 & $4 *$ \\
\hline Linearitätsfehler L in \%FS & $\leq 0,22$ & - \\
\hline $\begin{array}{l}\text { TK-Sensitivität TK-S in } \% \mathrm{~S}_{25}{ }^{\circ} \mathrm{C} / \mathrm{K} \\
\left(25^{\circ} \mathrm{C}-80^{\circ} \mathrm{C}\right)\end{array}$ & 0,08 & - \\
\hline
\end{tabular}

* Rechnerisch ermittelte Werte

\section{Schlussfolgerungen}

Die LTCC-Technologie weist eine Vielzahl von Eigenschaften auf, die sie für den Aufbau von piezoresistiven Kraftsensoren prädestiniert:

- Geeignet für dreidimensionale Mikrosysteme - chemisch und thermisch beständig,

- $\quad$ Lineares und nahezu hysteresefreies Materialverhalten,

- $\quad$ Einfache und kostengünstige Fertigung im Nutzen,

- Hausung der kraftempfindlichen Elemente im gleichen Materialsystem.

Die vorgestellten Kraftsensoren sind unter Verwendung der Grünfolie GT951 im Standard-LTCC-Prozess konzipiert. Die hohe Empfindlichkeit und der geringe Linearitätsfehler des Kraftsensors mit Kreisringplatte zeigen das Potential der Technologie für derartige Anwendungen. Die Weiterentwicklung findet sich in dem Kraftsensor mit Wagenradstruktur wieder. Hier sind eine deutliche Miniaturisierung und eine verbesserte Hausung umgesetzt.

Zukünftige Arbeiten beschäftigen sich mit der Charakterisierung der Wagenradstruktur für verschiedene Nennkraftbereiche, der Optimierung des Herstellungsprozesses und der Integration weiterer Funktionen wie z. B. Temperaturkompensation und dreidimensionale Kraftmessung.

\section{Literatur}

[1] U. Partsch, S. Gebhardt, D. Arndt, H. Georgi, H. Neubert, D. Fleischer, M. Gruchow: LTCC-Based Sensors for Mechanical Quantities. The $16^{\text {th }}$ European Microelectronics and Packaging Conference \& Exhibition. Proceedings. EMPC 2007, S. 381 - 388.

[2] M. S. Zarnik, D. Belavič, S. Maček: FE Analyses and Prototype Testing of a Piezoresistive LTCC-Based Low-Pressure Sensor. 15 International Symposium for Design Technology of Electronics Packages, SIITME 2009, S. 205 - 210.

[3] N. Craquelin, T. Maeder, Y. Fournier, P. Ryser: Low-cost-LTCC-based sensors for low force ranges. Proceedings of the Eurosensors XXIII conference, 2009, S. 899 - 902.

[4] G.Radosavljević, W. Smetana, A. Marić, Lj. Živanov, M. Unger, G.Stojanović: Micro Force Sensor Fabricated in the LTCC Technology. PROC. $27^{\text {th }}$ International Conference on Microelectonics, MIEL 2010 , S. 221 - 224.

[5] H. R. Tränkler, E. Obermeier: Sensortechnik. Handbuch für Praxis und Wissenschaft. Berlin, Heidelberg, New York: Springer Verlag 1998.

[6] K. W. Bonfig: Technische Druck- und Kraftmessung. Ethningen: Expert Verlag 1988. 